ISSN 0103-5150

Fisioter. Mov., Curitiba, v. 24, n. 4, p. 689-696, out./dez. 2011

Licenciado sob uma Licença Creative Commons

\title{
Efeito do isostretching no equilíbrio de indivíduos amputados: um estudo de caso
}

\author{
Effect of isostretching on balance of amputees: a case study
}

\author{
Marcos Willian Longato ${ }^{[a]}$, Priscilla Rodrigues de Castro ${ }^{[b]}$, Karon Cristine Keller ${ }^{[c]}$, \\ Danieli Isabel Romanovitch Ribas ${ }^{[\mathrm{d}]}$
}

[a] Acadêmico das Faculdades Integradas do Brasil (UniBrasil), Curitiba, PR - Brasil, e-mail: marcoslongato@hotmail.com

[b] Acadêmica das Faculdades Integradas do Brasil (UniBrasil), Curitiba, PR - Brasil, e-mail: pitcilla@hotmail.com

[c] Acadêmica das Faculdades Integradas do Brasil (UniBrasil), Curitiba, PR - Brasil, e-mail: karon@microcamponline.com.br

[d] Mestre em Tecnologia em Saúde, docente do Curso de Fisioterapia das Faculdades Integradas do Brasil (UniBrasil),

Curitiba, PR - Brasil, e-mail: danieliribas@yahoo.com.br

\section{Resumo}

Introdução: Para a manutenção do equilíbrio é necessária a integridade dos elementos anatômicos e funcionais. Por sua vez, a amputação de membros inferiores altera toda essa estrutura. 0 isostretching é uma técnica que promove o aumento da força muscular e melhora da conscientização postural, dos mecanismos proprioceptivos e do equilíbrio corporal. Objetivo: Avaliar os efeitos do isostretching no equilíbrio de um indivíduo amputado. Metodologia: Foi realizada avaliação do equilíbrio, pré e pós-aplicação de um programa de exercícios baseado no método isostretching, por meio da escala de Berg, de um indivíduo do sexo masculino, idoso, amputado transfemoral decorrente de trombose venosa profunda há um ano e sete meses, com uso de prótese há sete meses. Resultados: Ocorreu aumento na pontuação da escala de equilíbrio pós-aplicação do programa de exercícios propostos; porém, o equilíbrio estático e dinâmico do paciente permaneceu prejudicado. Conclusão: 0 efeito do isostretching causa melhora no equilíbrio de indivíduos amputados, mas o estudo não foi o suficiente para classificar o sujeito como livre de riscos de quedas.

Palavras-chave: Amputação. Equilíbrio. Isostretching. Fisioterapia. 
Abstract

Introduction: For the maintenance of balance, the integrity of anatomical and functional elements are necessary. The amputation of lower limb, however, affects all this structure. isostretching is a technique that promotes muscle strength, improves postural awareness, proprioceptive mechanisms and body balance. Objective: This study aims to evaluate the effects of isostretching on the balance of an amputee individual. Methods: The balance evaluation was made, before and after the application of a program of exercises based on isostretching method, through Berg Scale, of a male person, aged, with a transfemoral amputation due to deep vein thrombosis a year and seven months ago and who has been using prosthesis for seven months. Results: There was an increase in scores on the scale of balance after application of the proposed exercise program, however, the static and dynamic balance of the patient remained injured. Conclusion: Isostretching causes an improvement in the balance of amputees, but the study was not sufficient to classify the subject as free from falls risks.

Keywords: Amputation. Balance. Isostretching. Physical therapy.

\section{Introdução}

A amputação é um procedimento cirúrgico que, durante muito tempo, representou a única possibilidade de cirurgia para o homem. 0 termo designa a retirada de um órgão, ou parte dele, situado numa extremidade; porém, quando usado isoladamente, é entendido como amputação de membros (1).

$80 \%$ das amputações resultam de arteriopatias de etiologias diversas, das quais $46 \%$ seriam resultado de diabetes. Na ordem de porcentagem decrescente, seguem-se os casos de etiologia oncológica e, por último, os traumáticos (2).

A proporção de amputados de membros inferiores ocorre em maior número em diabéticos e fumantes, pacientes com história prévia de infarto do miocárdio e doenças vasculares cerebrais e pessoas que passam por acidente isquêmico (3). A amputação de membros inferiores é mais comum do que as de membros superiores, predomina no sexo masculino e em indivíduos jovens e é um procedimento cirúrgico que deixa sequelas irreversíveis (4).

Segundo Olarra (5), logo após a amputação, o indivíduo tende a apresentar quadros de sensação do membro fantasma, ao qual se relacionam dor ou sensibilidade na extremidade amputada.

Resende et al. (6), ao realizar um estudo com o objetivo de descrever a configuração da rede social de pessoas com amputação de membros, verificaram quem, quando um indivíduo perde um membro, ocorrem mudanças bruscas em sua vida e, consequentemente, o seu comportamento e maneira de agir são afetados diretamente. Esses indivíduos então têm como desafio ajustar-se psicologicamente à sua deficiência física para que a ela não se torne incapacitante.

Ao realizar um estudo referente ao tratamento ambulatorial de pacientes submetidos à amputação, Brito, Isernhagen e Depieri (7) observaram que a reabilitação após a amputação proporciona maior independência na realização de tarefas e prepara o paciente para a protetização. Gaspar (8) relata que pacientes possuem um menor gasto energético com a prótese em comparação com o uso de muletas.

O uso da prótese está estreitamente vinculado ao tipo da amputação, ou seja, as dificuldades impostas pela ausência de ambos os membros inferiores limitam o uso do membro artificial. Na amputação transfemural, é utilizada mais comumente a prótese composta de soquete com contato total quadrilateral, com ou sem sucção, podendo ser usado um cinto pélvico se o coto for muito próximo com apoio que se dá na região do ísquio, proporcionando a impressão de realizar a marcha como se estivesse sentado sobre ela. Na amputação transtibial, a prótese geralmente utilizada é a PTB com o pé SACH (solid ankle cushion heel - tornozelo sólido e calcanhar acolchoado), a qual permite contato total do coto, facilita seu uso, tem capacidade de apoio do peso sobre o tendão patelar e parece constituir a melhor prótese para esse tipo de amputação (9).

Baraúna (10) observou, em relação ao equilíbrio dos indivíduos amputados transtibiais, que ocorre uma correlação negativa entre medidas de oscilação anterior e idade, demonstrando que, à medida que o indivíduo atinge o domínio corporal, menores são as oscilações anteriores. Porém, nos amputados 
transfemurais protetizados, há maior tendência a oscilações anteriores, maiores oscilações no plano sagital comparados às oscilações no plano frontal. 0 tempo de uso da prótese também está diretamente relacionado às oscilações, pois, quanto maior o tempo de uso, mais o desequilíbrio tende a diminuir.

A postura corporal é uma resposta neuromecânica que se relaciona com a manutenção do equilíbrio, tendo a atividade muscular como importância para evitar a perda do equilíbrio a qual representa a atividade de controle automático da postura (11). Entre os métodos aplicados pela Fisioterapia no tratamento do equilíbrio, são citados pela literatura a equoterapia, a hidroterapia, o método isostretching, os exercícios vestibulares, entre outros. Porém, poucas são as referências de utilização desses métodos em indivíduos amputados.

Borges et al. (12) confirma que equoterapia contribui para melhora do equilíbrio de indivíduos amputados em membros inferiores, porém, por causa do pequeno número de amostra, são necessários mais estudos referentes ao assunto. De acordo com os estudos de Santos et al. (13), o protocolo de exercícios vestibulares propostos por Cawthorne e Cooksey resultam na melhora dos índices de queda apresentados por idosas avaliadas pela escala de Berg. Resende, Rassi e Viana (14) realizaram um estudo com o objetivo de avaliar o efeito de um programa de hidroterapia no equilíbrio e no risco de quedas em idosas, no qual avaliaram 25 idosas por meio da escala de Berg, Tinet e Time up \& Go; aplicaram um programa de exercícios de hidroterapia para equilíbrio e concluíram que a hidroterapia promove aumento significativo no equilíbrio de idosas e reduz, assim, o risco de quedas.

Sanglard (15), em seus estudos sobre a influência do isostretching nas alterações do equilíbrio de idosos, observou melhora no equilíbrio dos indivíduos submetidos ao treinamento. Esse resultado se deve ao aumento da força muscular, melhora da conscientização postural e dos mecanismos proprioceptivos proporcionados pela técnica.

0 método isostretching tem como objetivo realizar a sustentação corporal por meio de um reforço da musculatura profunda, da flexibilidade e da mobilidade muscular e articular, do controle respiratório e da concentração mental, proporcionando a manutenção e o controle do corpo no espaço. É suficientemente completo para flexibilizar as partes rígidas e fortalecer as debilitadas, solicitando o máximo de comandos musculares e induzindo ao cérebro a percepção das posturas corretas, assegurando, ainda, o controle da respiração e a participação ativa do indivíduo na distribuição de peso (16-18).

Carvalho e Assini (19) relatam que a contribuição do isostretching pode não estar ligada apenas ao aprimoramento da força muscular, mas, associadamente, à possibilidade de propiciar maior ativação $\mathrm{e}$ controle neuromuscular abdominal, melhorando o desempenho da marcha consequente à maior estabilização do tronco, sendo que essa estabilização espinhal é garantida pela interação entre três sistemas: o passivo (estrutura das vértebras, rigidez passiva dos discos, ligamentos espinhais, cápsula articular e propriedades passivas dos músculos); o ativo (propriedades contráteis dos músculos) e o sistema neural (proprioceptores e outros componentes neurais).

Levando em consideração que a amputação causa déficits em relação ao equilíbrio estático e dinâmico nos indivíduos amputados, e que os efeitos dos exercícios de isostretching nesse grupo ainda não são elucidados, é verificada a necessidade da realização deste estudo para melhor compreensão dos efeitos desses exercícios no equilíbrio dos indivíduos, permitindo, assim, o desenvolvimento de uma terapêutica adequada de tratamento.

\section{Materiais e métodos}

\section{Sujeitos}

Participou desse estudo um indivíduo, institucionalizado, do sexo masculino, com 67 anos, $95 \mathrm{~kg}$ e $178 \mathrm{~cm}$ de altura, sedentário, com amputação transfemural no membro inferior esquerdo decorrente de trombose venosa profunda há um ano e sete meses, fazendo uso de prótese há sete meses esporadicamente.

Para este estudo, foram utilizados como critério de inclusão amputação transfemural e uso de prótese no período mínimo de seis meses; e como critérios de exclusão, amputados bilaterais, alterações cognitivas, lesões de cerebelo, qualquer alteração musculoesquelética que prejudicasse realização dos exercícios propostos, e deficientes visuais e auditivos.

O presente estudo seguiu a Resolução 196/96 do Conselho Nacional de Saúde e foi aprovado pelo Comitê de Ética em Pesquisa das Faculdades Integradas do Brasil (UniBrasil) sob o parecer 15/2010. 
Procedimentos

Após a assinatura do Termo de Consentimento Livre e Esclarecido, o sujeito do estudo foi submetido à Avaliação do Equilíbrio pela escala de Berg Versão Brasileira (20), fazendo a utilização da prótese, antes e depois cinco semanas de aplicação de um programa de exercícios baseados no método isostretching.

A escala de Berg utiliza 14 tarefas que podem ser pontuadas de 0 a 4, o que totaliza o máximo de 56 pontos, que é a pontuação final máxima. Os scores de 0 a 20 correspondem a indivíduos que dependem de uma cadeira de rodas para locomoção. Scores de 21 a 40 indicam sujeitos que recorrem à assistência para locomoção, e scores de 41 a 56 correspondem a indivíduos que apresentam marcha independente (Quadro 1).

Por meio da escala de Berg também foi possível avaliar o risco de quedas. Na amplitude de 56 a 54, cada ponto a menos é associado a um aumento de $3 \%$ a $4 \%$ abaixo no risco de quedas; de 54 a 46 a alteração de um ponto é associada a um aumento de $6 \%$ a $8 \%$ de chances, sendo que abaixo de 36 pontos o risco de quedas é quase de $100 \%$ (Quadro 2) (21).

0 participante realizou os exercícios duas vezes por semana durante cinco semanas consecutivas e apresentou duas faltas, totalizando oito sessões. 0 programa de exercícios foi composto por seis posturas baseadas no método isostretching (três posturas em decúbito dorsal e três posturas sentada) com grau de

Quadro 1 - Avaliação da dependência

\begin{tabular}{lc}
\hline & Avaliação da dependência \\
\hline $0-20$ pontos & Dependente de cadeira de rodas \\
$21-40$ pontos & Necessitam de assistência na deambulação \\
$>41$ pontos & Marcha independente \\
\hline
\end{tabular}

Fonte: Adaptado de Miyamoto et al. (20).

Quadro 2 - Avaliação do risco de queda

\begin{tabular}{lc}
\hline \multicolumn{2}{c}{ Avaliação do risco de queda } \\
\hline 56 pontos & Pontuação máxima \\
$\mathbf{5 4 - 4 6}$ pontos & 6 a 8\% de risco de quedas \\
$<36$ pontos & $100 \%$ de risco de quedas \\
\hline
\end{tabular}

Fonte: Adaptado de Shumway-Cook et al. (21) dificuldade variado e sem a utilização da prótese. Foram realizadas três repetições para cada postura e, inicialmente, o sujeito foi orientado em relação à respiração preconizada pelo método. Os exercícios com bolas ou bastões foram utilizados como progressões.

No início e no final de cada sessão foi mensurada a pressão arterial e a frequência cardíaca para fins de controle.

Neste estudo, os grupos musculares preconizados de acordo com as posturas adotadas foram: quadríceps, reto abdominal, transverso abdominal, paravertebrais, tríceps braquial, grande dorsal, isquiotibiais, tríceps sural, glúteo médio, glúteo mínimo, o tensor da fáscia lata e as fibras superiores do glúteo máximo.

\section{Análise estatística}

Os dados coletados foram inseridos em planilhas e apresentados por meio de estatísticas descritivas e tabelas.

\section{Resultados}

A Tabela 1 apresenta os valores obtidos na avaliação do Equilíbrio do sujeito do estudo, por meio, da escala de Berg, pré e pós-aplicação do programa de exercícios baseados no método Isostretching.

De acordo com os resultados apresentados na Tabela 1 , na primeira avaliação, o indivíduo foi classificado, baseado na escala de Berg, como dependente de cadeira de rodas para locomoção. No entanto, após a aplicação dos exercícios, os resultados obtidos na reavaliação o classificaram como indivíduo que depende de auxílio para a deambulação. Foi observado também que, embora ocorra um aumento na pontuação após o programa de exercícios, o sujeito é classificado como suscetível a quedas, pois, de acordo com a escala de Berg, valores abaixo de 36 correspondem a

Tabela 1 - Pontuação adquirida pelo sujeito na pré e pósaplicação do protocolo de exercícios por meio da escala de Berg

\begin{tabular}{ccc}
\hline Avaliação berg & Pré-aplicação & Pós-aplicação \\
\hline Score total & 17 & 27 \\
\hline
\end{tabular}

Fonte: Dados da pesquisa. 
indivíduos que apresentam risco de quedas a quase de $100 \%$.

Na Tabela 2 são apresentados os valores obtidos pelo sujeito do estudo nas 14 tarefas preconizadas pela escala de Berg na pré-aplicação do programa de exercícios baseados no método isostretching. A Tabela 3 mostra a pontuação pós-aplicação do tratamento.

Ao comparar os resultados obtidos para cada atividade realizada, é possível perceber que, após a aplicação do programa de exercícios baseados no método isostretching, ocorreu aumento nos scores das habilidades avaliadas pela escala de Berg, exceto na posição de sentado sem apoio ao apanhar objeto no chão, virando-se para olhar pra trás e girando $360^{\circ}$, mantendo-se os scores.

\section{Discussão}

Os resultados obtidos no estudo demonstram que o paciente na primeira avaliação apresentava déficit de equilíbrio estático e dinâmico, o que o coloca em sérios riscos de queda. Miller, Speechley e Deathe (22) observam em seus estudos que indivíduos ido- sos que sofreram amputações de origem vasculares em membros inferiores apresentam maiores déficits de equilíbrio e maior insegurança, havendo maiores riscos de quedas. Além da amputação, Isquierdo (23) sugere que a idade causa a diminuição da força e da massa musculares, sendo estas associadas em parte com alterações ao equilíbrio. Faria et al. (24) descrevem que a função muscular tem sido fortemente relacionada aos eventos de quedas em pessoas e que, se houver treinamento, as quedas podem ser prevenidas.

0 método isostretching se propõe a realizar um fortalecimento da musculatura paravertebral profunda (25). A melhora do equilíbrio deve-se ao aumento da força muscular, à conscientização postural e aos mecanismos proprioceptivos proporcionados pela técnica (15).

Mesmo a pontuação na escala de Berg tendo mostrado uma melhora do score pós-aplicação dos exercícios de isostretching, é observado que os equilíbrios estático e dinâmico ainda permanecem prejudicados. Isso pode estar relacionado com o fato de o sujeito do estudo não utilizar a prótese com frequência. Baraúna (10) justifica que uso da prótese

Tabela 2 - Valores obtidos em cada atividade preconizada pela escala de Berg na pré-aplicação do isostretching

\begin{tabular}{|c|c|}
\hline Descrição dos itens & Pré-aplicação \\
\hline Sentado para em pé & $0=$ Necessitou de muita ajuda para se levantar. \\
\hline Em pé sem apoio & 2 = Conseguiu manter-se em pé, sem apoio durante 30 segundos. \\
\hline Sentado sem apoio & 4 = Manteve-se sentado com segurança e de forma estável durante dois minutos. \\
\hline Em pé para sentado & 1 = Sentou-se, de forma autônoma, mas sem controlar a descida. \\
\hline Transferências & $\begin{aligned} 0= & \text { Necessitou de duas pessoas para ajudar ou supervisionar de modo a transferir-se } \\
& \text { com segurança. }\end{aligned}$ \\
\hline Em pé com os olhos fechados & $2=$ Conseguiu manter-se em pé durante três segundos. \\
\hline Em pé com os pés juntos & $\begin{array}{l}1=\text { Necessitou de ajuda para chegar à posição, mas consegue manter-se em pé, } \\
\text { com os pés juntos, durante } 15 \text { segundos. }\end{array}$ \\
\hline Reclinar a frente com braços estendidos & $0=$ Perdeu o equilíbrio durante as tentativas / necessita de apoio externo. \\
\hline Apanhar objeto no chão & $3=$ Conseguiu apanhar o objeto, mas necessita de supervisão. \\
\hline Virando-se para olhar para trás & $1=$ Necessitou de supervisão ao virar. \\
\hline Girando $360^{\circ}$ & $0=$ Necessitou de ajuda enquanto dá a volta. \\
\hline Colocar os pés alternadamente sobre um banco & $0=$ Necessitou de ajuda para evitar a queda / não consegue tentar. \\
\hline Em pé com um pé em frente ao outro & $0=$ Perdeu o equilíbrio enquanto deu o passo e ao ficar de pé. \\
\hline Em pé apoiado em um dos pés & $\begin{array}{l}3=\text { Conseguiu levantar uma perna de forma autônoma e manteve a posição } \\
\text { durante sete segundos. }\end{array}$ \\
\hline Total & 17 \\
\hline
\end{tabular}

Fonte: Dados da pesquisa. 
Tabela 3 - Valores obtidos em cada atividade preconizada pela escala de Berg na pós-aplicação do isostretching

\begin{tabular}{|c|c|}
\hline Descrição dos itens & Pós-aplicação \\
\hline Sentado para em pé & 1 = Necessitou de alguma ajuda para se levantar ou manter estável. \\
\hline Em pé sem apoio & 3 = Conseguiu manter-se em pé durante dois minutos, com supervisão. \\
\hline Sentado sem apoio & 4 = Manteve-se igual à pontuação encontrada na pré aplicação. \\
\hline Em pé para sentado & $2=$ Apoiou a parte posterior das pernas na cadeira para controlar a descida. \\
\hline Transferências & 1 = Necessitou de ajuda de uma pessoa. \\
\hline Em pé com os olhos fechados & 3 = Conseguiu manter-se em pé durante 10 segundos, com supervisão. \\
\hline Em pé com os pés juntos & $\begin{array}{l}3=\text { Conseguiu manter os pés juntos, de forma autônoma e manteve-se em pé } \\
\text { durante um minuto, mas com supervisão. }\end{array}$ \\
\hline Reclinar a frente com braços estendidos & 1 = Inclinou-se para frente, mas necessitou de supervisão. \\
\hline Apanhar objeto no chão & 3 = Manteve-se igual à pontuação encontrada na pré-aplicação. \\
\hline Virando-se para olhar para trás & 1 = Manteve-se igual à pontuação encontrada na pré-aplicação. \\
\hline Girando $360^{\circ}$ & 0 = Manteve-se igual à pontuação encontrada na pré-aplicação. \\
\hline Colocar os pés alternadamente sobre um banco & 1 = Conseguiu completar mais de dois degraus, mas necessitou de ajuda. \\
\hline Em pé com um pé em frente ao outro & 0 = Manteve-se igual à pontuação encontrada na pré aplicação. \\
\hline Em pé apoiado em um dos pés & $\begin{array}{l}4=\text { Conseguiu levantar uma perna de forma autônoma e manter a posição durante } \\
15 \text { segundos. }\end{array}$ \\
\hline Total & 27 \\
\hline
\end{tabular}

Fonte: Dados da pesquisa.

está diretamente relacionado ao desequilíbrio, pois, quanto maior o tempo de uso, mais o desequilíbrio tende a diminuir.

A melhora do equilíbrio pós-aplicação do método isostretching pode estar relacionada com a melhora da percepção corporal, já que a amputação causa uma alteração brusca da imagem corporal, sendo importante que o indivíduo possa reformulá-la, pois essa perda da percepção corporal proporciona desequilíbrios e/ou quedas que acabam aumentando sentimentos de insegurança e fracasso no paciente em processo de reabilitação (26). Em estudo realizado para avaliar a percepção corporal por meio de exercícios de isostretching, Martins (27) relatou a melhora na tomada de consciência corporal comprovada pelo teste de Askevold (teste para verificação da consciência corporal) durante 10 sessões.

Em estudo realizado para verificar a influência do isostretching nas alterações de equilíbrio em idosos, Sanglard et al. (15) utilizaram como referência as escalas de Romberg, Romberg-Barré, Tinet, Berg e CAF. Foi observado resultado positivo em todos os testes realizados após a submissão aos exercícios programados e concluiu-se que o isostretching deve ser inserido no cotidiano dos indivíduos idosos, já que eles apresentam, como principal fator de queda, a diminuição do equilíbrio e da consciência corporal.

A pontuação encontrada no paciente classificando-o como suscetível a risco de quedas é explicada por Rubeinstein e Josephson (28), os quais afirmam que a incidência de quedas em idosos que residem na comunidade possui uma média de aproximadamente 0,7 quedas por ano. Já em idosos hospitalizados, a média sobe para aproximadamente 2,7 quedas; em idosos institucionalizados, a média é ainda maior, chegando a uma média de até 3,6 quedas por ano. Assim, como neste estudo, Miller et al. (22) comprovam que indivíduos que sofreram amputação transtibial e transfemural possuem alto risco de quedas.

Observou-se neste estudo que, após oito sessões de isostretching, houve uma melhora no desempenho funcional e motor e, por consequência, no equilíbrio do participante. Carvalho e Assini (19) apontam para novas evidências de que a capacidade funcional de sujeitos idosos pode ser aprimorada pela aplicação de exercícios baseados no método isostretching com melhora significativa a partir da quinta sessão.

0 aumento do score após a aplicação do protocolo do isostretching está relacionada com o uso do autocrecimento, contração isométrica (abdominais, 
glúteos, quadríceps e assoalho pélvico), alongamento muscular, além de uma expiração lenta e prolongada. Esse aumento desenvolve as capacidades musculares em desequilíbrio, fazendo com que ocorra a interação entre as estruturas anatômicas estáticas e dinâmicas na execução das atividades funcionais $(29,30)$.

Não foram encontrados outros estudos a respeito do uso de um protocolo de exercícios de isostretching em indivíduos amputados, portanto, houve dificuldade de encontrar parâmetros de comparação dos resultados.

\section{Conclusão}

De acordo com o presente estudo, foi possível verificar que os exercícios baseados no método isostretching podem minimizar as alterações de equilíbrio nos indivíduos amputados, promovendo uma melhora no desenvolvimento funcional e motor.

Novos estudos devem ser realizados com amostra maior, a fim de comprovar a eficácia do método isostretching no equilíbrio dessa população e de verificar o efeito desse método por meio do aumento do número de sessões e pós-descondicionamento.

\section{Referências}

1. Chini GCO, Boemer MR. Amputação na percepção de quem a vivencia: um estudo sob a ótica fenomenológica. Rev Latino-Am Enfermagem. 2007;15(2):330-6. doi: 10.1590/S0104-11692007000200021.

2. Gabriel RS, Petit JD, Carril LS. Fisioterapia em traumatologia ortopedia e reumatologia. Rio de Janeiro: Revinter; 2001.

3. Perales MC, Cortés MJG, Utiel GV, Gil JM, Peres del Barrio P, Hinojosa JB. Incidencia y factores de riesgo de amputación no traumática de miembros inferiores en los pacientes en hemodiálisis. Nefrología. 2005; 25(4):399-406.

4. Cassefo V, Nacaratto DC, Chamlian TR. Perfil epidemiológico dos pacientes amputados do Lar Escola São Francisco: estudo comparativo de 3 períodos diferentes. Acta Fisiátrica. 2003;10(2):67-71.

5. Olarra J, Longarela A. Sensación de miembro fantasma y dolor de miembro residual tras 50 años de la amputación. Rev Soc Esp del Dolor. 2007;14(6):428-31.
6. Resende MC, Cunha CPB, Silva AP, Souza SJ. Rede de relações e satisfação com a vida em pessoas com amputação de membros. Ciências \& Cognição. 2004; 10(1):164-77.

7. Brito DD, Isernhagen FC, Depieri TZ. Tratamento fisioterapêutico ambulatorial em paciente submetido à amputação transfemoral unilateral por acidente motociclístico: estudo de caso. Arq Ciênc Saúde Unipar. 2005;9(3):175-80.

8. Gaspar AP, Ingham SJN, Chamlian TR. Gasto energético em paciente amputado transtibial com prótese e muletas. Acta Fisiátrica. 2003;10(1):32-4.

9. Diogo MJD’E. Avaliação funcional de idosos com amputação de membros inferiores atendidos em um hospital universitário. Rev Latino-Am Enfermagem. 2003; 11(1):59-65.

10. Baraúna MA, Duarte F, Sanchez HM, Canto RST, Malusá S, Campelo-Silva CD, et al. Avaliação do equilíbrio estático em indivíduos amputados de membros inferiores por meio da biofotogrametria computadorizada. Rev Bras Fisioter. 2006;10(1):83-90.

11. Enoka RM. Bases neuromecânicas da cinesiologia. São Paulo: Manole; 2000.

12. Borges T, Lemos LFC, Araújo R, Santana LA, Lopes M, Franck CR. Equoterapia para melhora do equilíbrio postural em amputados de membro inferior: um estudo piloto. Revista Digital. 2009 [acesso 18 set. 2010]; 14(135). Disponível em: www.efdeportes.com.

13. Santos AC, Ferreira CP, Silva KC, Araújo VV, Fernandes C. Exercícios de Cawthorne e Cooksey em idosas: melhora do equilíbrio. Fisioter Mov. 2008;21(4):129-36.

14. Resende SM, Rassi CM, Viana FP. Efeitos da hidroterapia na recuperação do equilíbrio e prevenção de quedas em idosos. Rev Bras Fisioter. 2008;12(1):57-63.

15. Sanglard RCF, Pereira JS, Henriques GRP, Gonçalves GB. A influência do isostretching nas alterações do equílibrio em idosos. Rev Bras Ciênc Mov. 2007;15(2): 63-71.

16. Bonorino KC, Borin GS, Silva AH. Tratamento para escoliose por meio do método iso stretching e uso de bola suíça. Cinergis. 2007;8(2):1-6.

17. Redondo B. Isostretching: a ginástica da coluna. Piracicaba: Skin Direct Store; 2001. 
18. Oliveiras AP, Souza DE. Tratamento fisioterapêutico em escoliose por meio das técnicas de Iso-Stretching e manipulações osteopáticas. Terap Man. 2004;2(3): 104-13.

19. Carvalho AR, Assini TCKA. Improvement of functional capacity among elderly people undergoing isostretching intervention. Rev Bras Fisioter. 2008; 12(4):268-73.

20. Miyamoto ST, Lombardi I Jr, Berg KO, Ramos LR, Natour J. Brazilian version of the Berg balance scale. Braz J Med Biol Res. 2004;37(9):1411-21.

21. Shumway-Cook A, Baldwin M, Polissar NL, Gruber W. Predicting the probability for falls in communitydwelling older adults. Phys Ther. 1997;77(8):812-9.

22. Miller WC, Mark S, Deathe AB. Balance confidence among people with lower-limb amputations. Phys Ther. 2002;82(9):856-65.

23. Izquierdo $M$, Ibañez J, Gorostiaga $E$, Garrues $M$, Zúñiga A, Antón A Larrión JL, et al. Maximal strength and power characteristics in isometric and dynamic actions of the upper and lower extremities in middleaged and older men. Acta Physiol Scand. 1999;167(1): 57-68.

24. Faria JC, Machala CC, Dias RC, Dias JMD. Importância do treinamento de força na reabilitação da função muscular, equilíbrio e mobilidade de idosos. Acta Fisiátrica. 2003;10(3):133-7.
25. Durante H, Vasconcelos ECLM. Comparação do método isostretching e cinesioterapia convencional no tratamento da lombalgia. Semina: Ciências Biológicas e da Saúde. 2009;30(1):83-90.

26. de Benedetto KM, Forgione MCR, Alves VLR. Reintegração corporal em pacientes amputados e a dorfantasma. Acta Fisiátrica. 2002;9(2):85-9.

27. Martins RO. A prática do iso-stretching na melhora da percepção corporal [monografia]. Cascavel: Unioeste; 2004.

28. Rubeinstein LZ, Josephson KR. The epidemiology of falls and syncope. Clin Geriatr Med. 2002;18(2):141-58.

29. Macedo CSG, Debiagi PC, Andrade FM. Efeito do isostretching na resistência muscular de abdominais, glúteo máximo e extensores de tronco, incapacidade e dor em pacientes com lombalgia. Fisioter Mov. 2010; 23(1):113-20.

30. Prado ALC, Fonseca PHS, Rodrigues CO, Vanz F. O método Iso-stretching na otimização das aptidões para a prática do futebol de campo. Rev Saúde. 2004; 30 (1/2):57-64.

Recebido: $11 / 11 / 2010$

Received: 11/11/2010

Aprovado: 24/05/2011

Approved: 05/24/2011 